\title{
In vitro growth of Plasmodium falciparum in neonatal blood
}

\author{
Ulrich Sauerzopf ${ }^{1,2}$, Yabo J Honkpehedji², Ayôla A Adgenika 2,3,4, Elianne N Feugap², Ghyslain Mombo Ngoma2,3, \\ Jean-Rodolphe Mackanga², Felix Lötsch ${ }^{1,2}$, Marguerite M Loembe ${ }^{2,3,5}$, Peter G Kremsner ${ }^{2,3}$, Benjamin Mordmüller ${ }^{2,3}$ \\ and Michael Ramharter ${ }^{1,2,3^{*}}$
}

\begin{abstract}
Background: Children below the age of six months suffer less often from malaria than older children in sub-Saharan Africa. This observation is commonly attributed to the persistence of foetal haemoglobin ( $\mathrm{HbF})$, which is considered not to permit growth of Plasmodium falciparum and therefore providing protection against malaria. Since this concept has recently been challenged, this study evaluated the effect of $\mathrm{HbF}$ erythrocytes and maternal plasma on in vitro parasite growth of $P$. falciparum in Central African Gabon.

Methods: Umbilical cord blood and peripheral maternal blood were collected at delivery at the Albert Schweitzer Hospital in Gabon. Respective erythrocyte suspension and plasma were used in parallel for in vitro culture. In vitro growth rates were compared between cultures supplemented with either maternal or cord erythrocytes. Plasma of maternal blood and cord blood was evaluated. Parasite growth rates were assessed by the standard HRP2-assay evaluating the increase of HRP2 concentration in Plasmodium culture.

Results: Culture of $P$. falciparum using foetal erythrocytes led to comparable growth rates (mean growth rate $=4.2$, $95 \% \mathrm{Cl}: 3.5-5.0$ ) as cultures with maternal red blood cells (mean growth rate $=4.2,95 \% \mathrm{Cl}: 3.4-5.0$ ) and those from non-malaria exposed individuals (mean growth rate $=4.6,95 \% \mathrm{Cl}: 3.8-5.5$ ). Standard in vitro culture of $P$. falciparum supplemented with either maternal or foetal plasma showed both significantly lower growth rates than a positive control using non-malaria exposed donor plasma.

Conclusions: These data challenge the concept of $\mathrm{HbF}$ serving as intrinsic inhibitor of $P$. falciparum growth in the first months of life. Erythrocytes containing $\mathrm{HbF}$ are equally permissive to $P$. falciparum growth in vitro. However, addition of maternal and cord plasma led to reduced in vitro growth which may translate to protection against clinical disease or show synergistic effects with $\mathrm{HbF}$ in vivo. Further studies are needed to elucidate the pathophysiology of innate and acquired protection against neonatal malaria.
\end{abstract}

Keywords: Plasmodium falciparum, Neonatal haemoglobin, Malaria, Histidine-rich protein 2, Immune plasma

\section{Background}

Although children below five years of age are disproportionally severely affected by malaria morbidity and mortality, there is a lag time of about three months after birth, before first disease episodes occur [1-4]. This period coincides with the persistence of maternal IgG antibodies in the infant's circulation but other protective

\footnotetext{
* Correspondence: michael.ramharter@medizin.uni-tuebingen.de

${ }^{1}$ Department of Medicine I, Division of Infectious Diseases and Tropical

Medicine, Medical University of Vienna, Vienna, Austria

${ }^{2}$ Centre de Recherches Médicales de Lambaréné, Hôpital Albert Schweitzer,

Lambaréné, Gabon

Full list of author information is available at the end of the article
}

mechanisms may also contribute to the reduced susceptibility [4-7].

Previous studies postulate a role for persisting foetal haemoglobin during the first months of life in protection against malaria [8]. The transcription of $\mathrm{HbF}$ starts around the 10th week of development and ends shortly before birth. This causes a linear decline in the number of foetal red blood cells from up to $90 \%$ at time of birth to around 5\% at 3 months of age [8]. It was hypothesized that parasite strains - encountering $\mathrm{HbF}$ only on relatively rare occasions from an evolutionary perspective may therefore be selected by strong adaptation to adult 
haemoglobin $(\mathrm{HbA})$, a process resulting in limited intraerythrocytic growth of Plasmodium falciparum in predominantly HbF containing neonatal blood $[9,10]$. This paradigm of protection during early infancy - first published in 1977 - was held up since then until recently, when Amaratunga et al. failed to detect growth delays in neonatal erythrocytes and thus profoundly challenged this concept [11].

To further investigate potential mechanisms of protection, this study evaluated the comparative in vitro growth rates of a standardized $P$. falciparum clone under controlled culture conditions using either maternal, cord, or non-malaria exposed donor erythrocytes.

In addition, the growth modulating effect of maternal, cord, and non-malaria exposed donor plasma on P. falciparum growth was evaluated.

\section{Methods}

\section{Study region and patient population}

The study took place at the Centre de Recherches Médicales de Lambaréné, Albert Schweitzer Hospital and Georges Rawiri Regional Hospital in Lambaréné, Gabon. Gabon is a Central African country characterized by a tropical climate and hyperendemic malaria transmission [12]. Participants were invited to join this study when attending the local maternity wards for delivery. Demographic data were obtained, however no validated information about intake of IPTp was available. All subjects were tested in routine antenatal care for HIV and only those with negative test result were invited to participate. The study protocol was approved by the institutional review board of the CERMEL and the ethical review committee of the Medical University of Vienna and all women provided written informed consent prior to blood sampling.

All samples were tested for sickle cell trait by haemoglobin electrophoresis and for plasmodial infection by thick smear at the time of delivery and samples were excluded in case of a positive result or when signs of haemolysis or clotting were apparent. At delivery $1.2 \mathrm{ml}$ of blood was taken from the umbilical cord in an EDTA tube. Peripheral maternal venous blood was taken at the earliest convenience within three days. Non-malaria exposed donor blood was obtained from Caucasian male volunteers. Blood samples were collected in EDTA tubes and specimens were immediately centrifuged to separate red blood cells from plasma. Red blood cells were washed three times in complete parasite medium and stored at $4^{\circ} \mathrm{C}$ in 0.5 volume of saline adenine glucose-mannitol $(150 \mathrm{mM} \mathrm{NaCl}, 1.25 \mathrm{mM}$ adenine, $45 \mathrm{mM}$ glucose, $30 \mathrm{mM}$ mannitol) until further use. Plasma was immediately frozen at $-80^{\circ} \mathrm{C}$.

\section{Parasite culture and growth assay}

A laboratory-adapted clone of P. falciparum (3D7) repeatedly selected for presence of knob-phenotype was kept in continuous sorbitol synchronized culture throughout the course of the study. Parasites were maintained using a standard protocol in complete parasite medium (500 ml RPMI-1640, $10 \mathrm{mg} / \mathrm{l}$ gentamicin, $6 \mathrm{~g} / \mathrm{l}(25 \mathrm{mM})$ HEPES, $292 \mathrm{mg} / \mathrm{l}$ (2 mM) L-glutamine, $50 \mathrm{mg} / \mathrm{l}(0.36 \mathrm{mM})$ hypoxanthine, $5 \mathrm{~g} / \mathrm{l}$ Albumax II) in a candle jar at $37^{\circ} \mathrm{C}$. To test the effect of erythrocytes and plasma on plasmodial growth, micro-cultures of $200 \mu \mathrm{l}$ each were established in 96-well plates (Corning Costar-3599) in duplicates. To investigate the effect of $\mathrm{HbF}$ on parasite growth, microcultures containing cord derived red blood cells and maternal red blood cells, respectively, as well as a positive control containing donor $\mathrm{O}+$ red blood cells, were set up in duplicates. Parasites derived from continuous culture in adult $\mathrm{RBC}$ were diluted with respective test $\mathrm{RBC}(>1: 40)$ to obtain at $1.5 \%$ haematocrit, $0.05 \%$ parasitaemia using $5 \%$ pooled, heat inactivated serum from $\mathrm{AB}+$ donors for 72 hours before growth was stopped by freezing.

Plasma derived from mothers, newborns and a malarianaïve adult was added to cultures in complete parasite medium to assess its effect on parasite growth at 1.5\% haematocrit, $0.05 \%$ parasitaemia and $10 \%$ plasma supplementation. Frozen lysates of micro-cultures were used for histidine-rich protein II enzyme linked immunosorbent assay (HRP2 ELISA) for the quantification of parasite growth [13-15]. Commercial antibodies (MPFM-55A and MPFG-55P, Immunology Consultants Laboratories, Inc) and high binding plates (Corning Costar 3590) were used. Growth rates of $P$. falciparum were estimated by the assessment of HRP2 concentrations in cultures. Growth was defined as an increase in optical density in the HRP2 ELISA. An at least two fold increase in HRP2 concentration from prior to post incubation was set as threshold for further analysis. Statistical analysis was performed using " $R$ " software. Differences between groups were assessed employing the Tukey's Honestly Significant Difference test. All reagents, unless stated otherwise were obtained from Sigma-Aldrich, St. Louis.

\section{Results}

Hundred and three pregnant women consented to participate in this study between July 2012 and June 2013, of which sixty-five were excluded from further testing (sickle cell trait: 12, clotting: 27, haemolysis after sampling: 13, Plasmodium infection: 7, presence of antiRhesus antibodies: 6). Thirty-eight participants finally met the criteria for analysis. Mother's age was ranging from 17 to 42 years with a mean of 25 years and a standard deviation of 7.1 years with a mean gravidity of 3.5 with a standard deviation of 2.4. Mean gestational age at delivery was 38.5 weeks of gestation with a standard deviation of 2.1 weeks and mean birth weight was 2,866 grams ranging from 1,900 to 4,400 grams. Among the recruited women, 18 paired erythrocyte samples and 22 


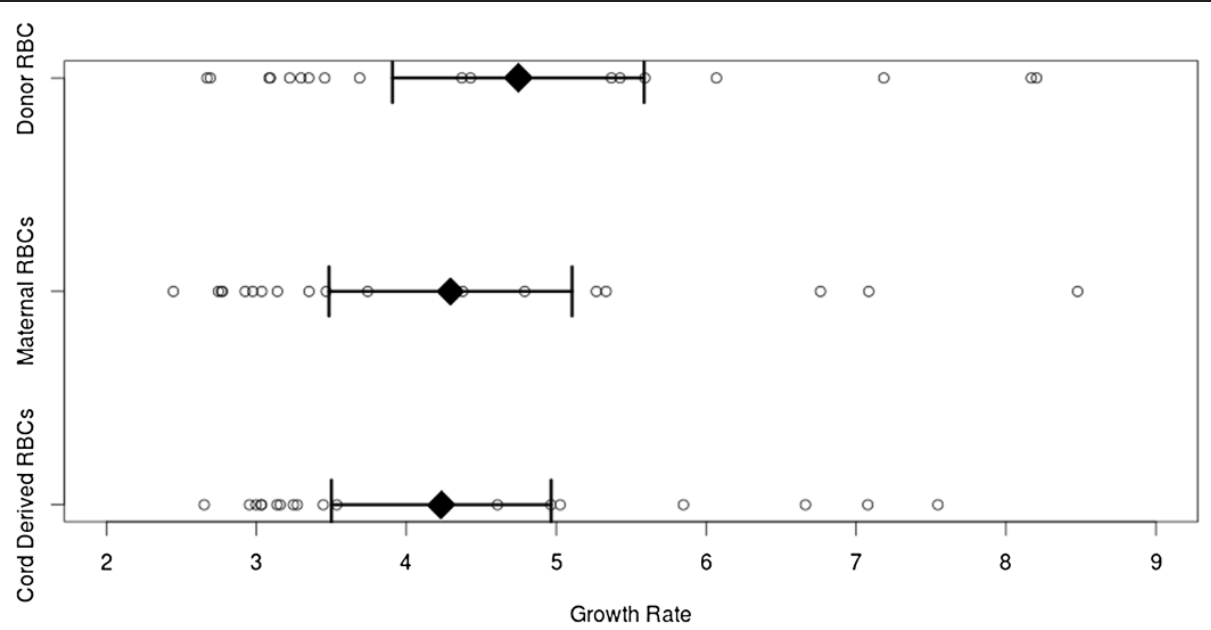

Figure 1 In vitro growth rates of Plasmodium falciparum when using cord, maternal or non-malaria exposed donor erythrocytes displayed by data points, means and $95 \% \mathrm{Cls}$.

plasma specimens were successfully used in parallel for further in vitro testing.

\section{Maternal, neonatal, and non-malaria exposed donor erythrocytes}

Parasite growth was quantified by HRP2 ELISA. 18 paired samples of maternal, cord and donor RBCs showed adequate growth and were used for comparative evaluation. Mean cumulative growth rates were 4.35 (95\% confidence interval: 3.90 - 4.81) folds when compared with preincubation samples. No significant difference between cultures using non-malaria exposed donor erythrocytes (mean growth rate: $4.6,95 \% \mathrm{CI}: 3.8-5.5$ ) or either maternal $(4.2,95 \%$ CI: $3.4-5.0)$ or cord derived erythrocytes (4.2, 95\% CI: 3.5 - 5.0) was observed (Figure 1). Similarly, growth rates between maternal and cord derived erythrocytes supplemented cultures did not differ $(\mathrm{p}=0.99)$. To test these findings under different culture conditions, cultures were classified in high growth ( $>4$ fold increase) and low growth strata (2-4 fold increase in HRP2). When comparing growth rates within these strata, again no significant difference was observed (Table 1).

In addition to this quantitative analysis, a qualitative analysis of intra-erythrocytic growth of $P$. falciparum was performed microscopically. Parasites growing in umbilical cord erythrocytes displayed microscopically similar maturation characteristics and no other obvious morphological differences were observed (Figure 2).

\section{Maternal, neonatal, and non-malaria exposed plasma}

Growth rates of $P$. falciparum were compared under standard conditions by supplementing culture medium with either maternal or cord derived plasma and nonmalaria exposed donor plasma serving as a positive control. Plasmodium falciparum growth was significantly higher in non-malaria exposed donor plasma containing culture medium than in either culture supplemented with test plasma (Figure 3). Whereas the mean increase in HRP2 concentration in culture supplemented with malaria-naïve plasma was 6.8 fold, growth rates in cultures supplemented with maternal and umbilical cord plasma averaged 2.1 fold ( $\mathrm{p}<0.001$ ). Umbilical cord plasma (mean growth rate: 2.1, 95\% CI: 2.0 - 2.3) and maternal plasma (mean growth rate: 2.1, 95\% CI: 2.0 $2.2)$ yielded close to identical results. $(p=0.93)$

\section{Discussion}

This study demonstrates that cord derived erythrocytes, harbouring a high concentration of $\mathrm{HbF}$, are equally suited for $P$. falciparum invasion, maturation, and growth as adult erythrocytes containing predominantly

Table 1 Mean growth rates and lower and upper margin of $95 \% \mathrm{Cls}$ in test given test erythrocytes of high and low growth strata as well as the cumulative data

\begin{tabular}{lccc}
\hline All data & $\begin{array}{c}\text { Lower } \\
\text { margin }\end{array}$ & Mean & $\begin{array}{c}\text { Upper } \\
\text { margin }\end{array}$ \\
\hline Cord derived RBC & 3.50 & 4.23 & 4.97 \\
Maternal RBC & 3.38 & 4.19 & 5.00 \\
Non malaria exposed donor RBC & 3.79 & 4.63 & 5.47 \\
Low growth & & & \\
Cord derived RBC & 4.63 & 5.36 & 6.08 \\
Maternal RBC & 4.63 & 5.43 & 6.23 \\
Non malaria exposed donor RBC & 5.42 & 6.09 & 6.76 \\
High growth & & & \\
Cord derived RBC & 3.01 & 3.11 & 3.21 \\
Maternal RBC & 2.81 & 2.95 & 3.10 \\
Non malaria exposed donor RBC & 3.02 & 3.17 & 3.33 \\
\hline
\end{tabular}




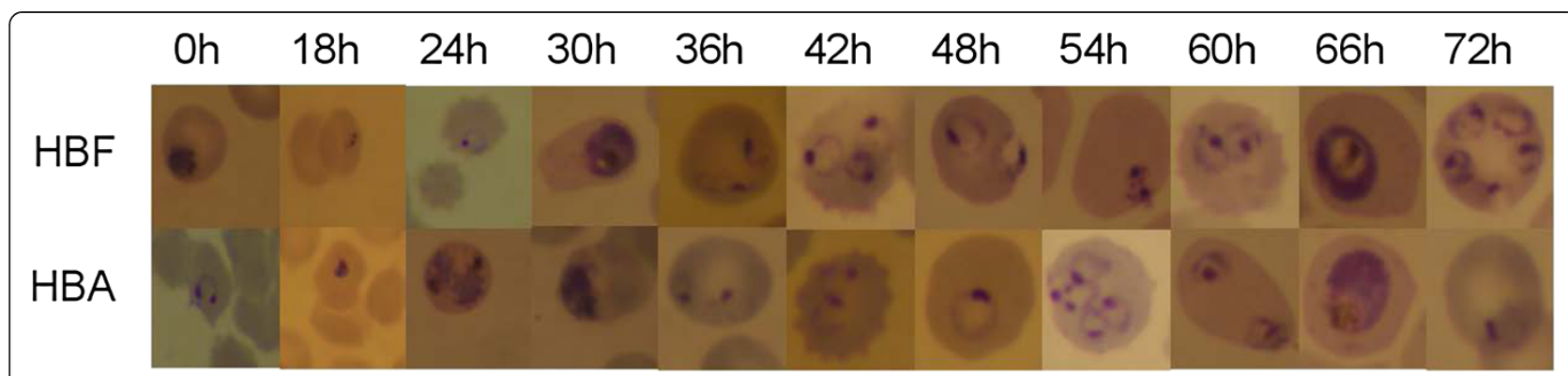

Figure 2 In-vitro development of Plasmodium falciparum over time in erythrocytes rich in foetal and adult haemoglobin respectively.

HbA. Growth rates between maternal, neonatal and non-malaria exposed donor erythrocytes were very similar, and no morphological signs of growth inhibition were detected. These findings provide strong evidence that P. falciparum may similarly develop within $\mathrm{HbF}$ rich red blood cells and that $\mathrm{HbF}$ per se is no protective factor against malaria during the first months of life.

These data are in line with results published by Amaratunga et al. [11] demonstrating normal invasion and development of $P$. falciparum in $\mathrm{HbF}$ containing red blood cells in a set of adult and six cord blood specimens. While this study focused on an overall assessment of parasite growth in a larger sample size, Amaratunga et al. could detect effects of neonatal haemoglobin, in particular in conjunction with immune IgGs, on the knob phenotype and PfEMP-1 mediated cytoadherence of parasitized red blood cells. Although erythrocytes rich in foetal haemoglobin seem to be permissive to infection with $P$. falciparum in vitro, reduced cytoadherence may, particularly in the presence of IgG, lead to lower parasite densities in vivo due to increased clearance of infected cells in the spleen.

To evaluate whether humoral immunity and transfer of maternal immunoglobulins or other plasmatic factors may play a protective role in newborns, cultures were supplemented with the respective plasma samples. Interestingly, a consistent growth-inhibition was observed in both maternal and neonatal plasma when compared to non-malaria exposed donor plasma. In vitro growth inhibition caused by a supplementation with immune plasma was reported previously in Thai individuals yielding comparable results to this study [16].

It is tempting to speculate that passive transfer of maternal antibodies to the newborn accounts for this growth inhibitory effect. However, it may not be excluded that other factors including cytokines, hormones, complement or indeed drugs with anti-malarial activity passing the placental barrier into the neonatal blood may have caused this inhibition of growth. One limitation of this study is the lack of valid information about IPTp intake of study participants - a factor potentially affecting plasma assays. In addition these data derive from experiments with one clone of P. falciparum and potential differences between wild isolates and laboratory adapted parasites may not be ruled out. Finally, behavioural factors certainly also contribute to young infants' protection from malaria, since neonates receive special care and attention by their caregivers potentially leading to less exposure to infectious bites.

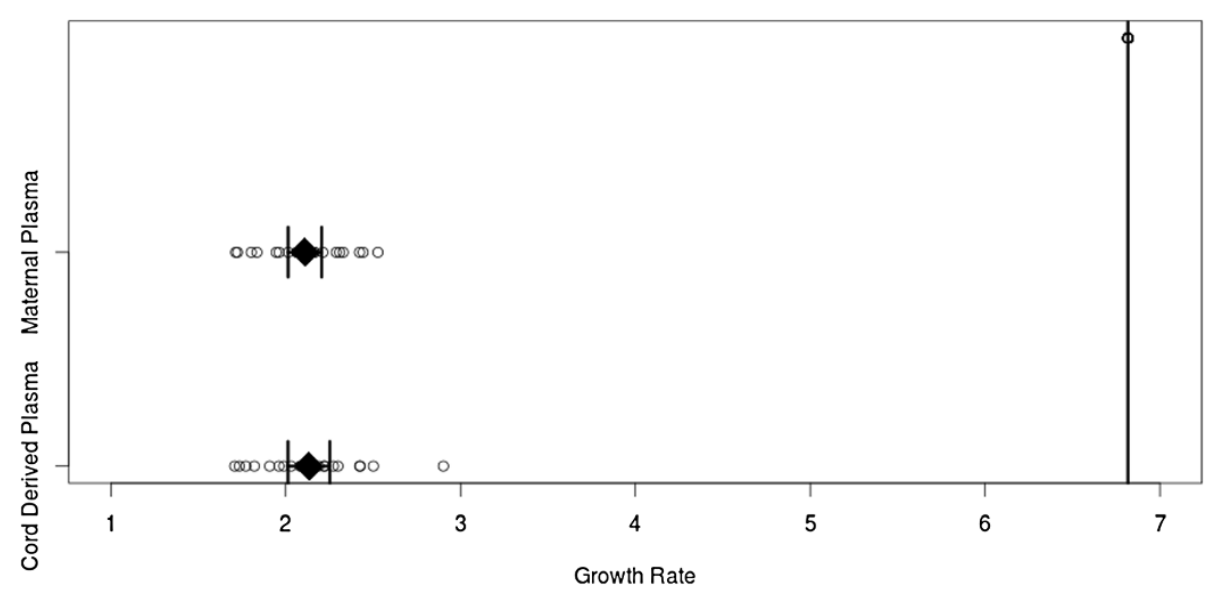

Figure 3 In vitro growth rates of Plasmodium falciparum when supplemented with either cord or maternal plasma displayed by data points, means and $95 \%$ Cls. Non-malaria exposed donor plasma used as a positive control yielded a growth rate of 6.8 , indicated by the vertical bar. 
The amount of protection against malaria in early infancy might yet be caused by the interplay of several distinct factors, probably in conjunction with maternally derived antibodies. However, these data conclusively refute the hypothesis of $\mathrm{HbF}$ serving as an intrinsic inhibitor of $P$. falciparum growth in vitro, and indicate that maternal and newborn plasma show considerable inhibitory activity against in vitro growth of $P$. falciparum.

\section{Competing interests}

None of the authors declares a conflict of interest. MR is member of the Editorial Board of the Malaria Journal.

\section{Authors' contributions}

$\mathrm{BM}, \mathrm{MR}$, and US developed the study design. MR and US conceived the analyses and drafted the manuscript. AAA, NFE and HJ recruited study participants. US conducted laboratory analysis. MML MR and AAA participated in study conduct and provided logistical assistance. All authors read and approved the final manuscript.

\section{Acknowledgements}

We thank all nurses and midwifes at the Albert Schweitzer Hospital and Georges Rawirii Regional Hospital in Lambaréné for their kind cooperation. This work was supported by Deutsche Forschungsgemeinschaft (DFG-Projekt KR 1150/6-1) and Open Access Publishing fund of the University of Tübingen, and by EU-funded project Immunological Interplay between Poverty Related Diseases and Helminth infections: An African-European Research Initiative "IDEA" (HEALTH-F3-2009-241642). We are grateful for the logistic and infrastructure support kindly provided by CANTAM.

\section{Author details}

${ }^{1}$ Department of Medicine I, Division of Infectious Diseases and Tropical Medicine, Medical University of Vienna, Vienna, Austria. ${ }^{2}$ Centre de Recherches Médicales de Lambaréné, Hôpital Albert Schweitzer, Lambaréné, Gabon. ${ }^{3}$ Institut für Tropenmedizin, Universität Tübingen, Wilhelmstraße 27, 72074 Tübingen, Germany. ${ }^{4}$ Department of Parasitology, Leiden Medical University Center, Leiden, The Netherlands. ${ }^{5}$ Faculté de Médecine et des Sciences de la Santé, Université Omar Bongo, Libreville, Gabon.

Received: 10 August 2014 Accepted: 3 November 2014

Published: 18 November 2014

\section{References}

1. WHO: World Malaria Report. Geneva: World Health Organization; 2013.

2. Greenwood BM, Bojang K, Whitty CJ, Targett GA: Malaria. Lancet 2005, 365:1487-1498.

3. Klein Klouwenberg PMC, Oyakhirome S, Schwarz NG, Gläser B, Issifou S, Kiessling G, Klöpfer A, Kremsner PG, Längin M, Lassmann B, Necek M, Pötschke M, Ritz A, Grobusch MP: Malaria and asymptomatic parasitaemia in Gabonese infants under the age of 3 months. Acta Trop 2005, 95:81-85.

4. Ramharter M, Schuster K, Bouyou-Akotet MK, Adgenika AA, Schmits K, Mombo-Ngoma G, Agnandji ST, Nemeth J, Afène SN, Issifou S, Onnas IN, Kombila M, Kremsner PG: Malaria in pregnancy before and after the implementation of a national IPTp program in Gabon. Am J Trop Med Hyg 2007, 77:418-422.

5. Riley EM, Wagner GE, Akanmori BD, Koram KA: Do maternally acquired antibodies protect infants from malaria infection? Parasite Immunol 2001, 23:51-59.

6. Bardají A, Sigauque B, Laia B, Romagosa C, Sanz S, Mabunda S, Mandomando I, Aponte J, Sevene E, Alonso PL, Menéndez C: Clinical malaria in African pregnant women. Malar J 2008, 7:27.

7. Ramharter M, Grobusch MP, Kießling G, Adegnika AA, Möller U, Agnandji STM, Kramer M, Schwarz N, Kun JFJ, Oyakhirome S, Issifou S, Borrmann S, Lell B, Mordmüller B, Kremsner PG: Clinical and parasitological characteristics of puerperal malaria. J Infect Dis 2005, 191:1005-1009.

8. Wilson RJM, Pasvol G, Weatherall DJ: Invasion and growth of Plasmodium falciparum in different types of human erythrocytes. Bull World Health Organ 1977, 55:79-186.
9. Colombo B, Kim B, Atencio RP, Molina C, Terrenato L: The pattern of fetal haemoglobin disappearance after birth. Br J Haematol 1976, 32:79-87.

10. Pasvol G, Weatherall DJ, Wilson RJM: Effects of foetal haemoglobin on susceptibility of red cells to Plasmodium falciparum. Nature 1977, 270:171-173.

11. Amaratunga C, Lopera-Mesa TM, Brittain NJ, Cholera R, Arie T, Fujioka $H_{\text {, }}$ Keefer JR, Fairhurst RM: A role for fetal hemoglobin and maternal immune IgG in infant resistance to Plasmodium falciparum malaria. PLoS One 2011, 6:e14798.

12. Ramharter M, Adegnika AA, Agnandjil ST, Matsiegui PB, Grobusch MP, Winkler S, Graninger W, Krishna S, Yazdanbakhsh M, Mordmüller B, Lell B, Missinou MA, Mavoungou E, Issifou S, Kremsner PG: History and perspectives of medical research at the Albert Schweitzer Hospital in Lambaréné, Gabon. Wien Klin Wochenschr 2007, 119:8-12.

13. Noedl H, Bronnert J, Yingyuen K, Attlmayr B, Kollaritsch H, Fukuda M: Simple histidine-rich protein 2 double-site sandwich enzyme-linked immunosorbent assay for use in malaria drug sensitivity testing. Antimicrob Agents Chemother 2005, 49:3575-3577.

14. Ramharter M, Noedl H, Thimasarn K, Wiedermann G, Wernsdorfer G, Wernsdorfer $\mathrm{WH}$ : In vitro activity of tafenoquine in combination with artemisinin against Plasmodium falciparum. Am J Trop Med Hyg 2002, 67:39-43.

15. Ramharter M, Wernsdorfer WH, Kremsner PG: In vitro activity of quinolines against Plasmodium falciparum in Gabon. Acta Trop 2004, 90:55-60.

16. Monatrakul $P$, Mungthin M, Dondorp AM, Krudsood S, Udomsangpetch R, Wilairatana P, White NJ, Chotivanich K: Modulating effects of plasma containing anti-malarial antibodies on in vitro anti-malarial drug susceptibility in Plasmodium falciparum. Malar J 2010, 9:326.

doi:10.1186/1475-2875-13-436

Cite this article as: Sauerzopf et al:: In vitro growth of Plasmodium falciparum in neonatal blood. Malaria Journal 2014 13:436.

\section{Submit your next manuscript to BioMed Central and take full advantage of:}

- Convenient online submission

- Thorough peer review

- No space constraints or color figure charges

- Immediate publication on acceptance

- Inclusion in PubMed, CAS, Scopus and Google Scholar

- Research which is freely available for redistribution 